\title{
Isolation, identification, and antioxidant activity of polysaccharides from the shell of abalone (Haliotis discus hannai Ino)
}

\author{
Z.L. Wang ${ }^{1 *}$, H.B. Liang ${ }^{1 *}$, W. Guo ${ }^{1 *}$, Z.F. Peng ${ }^{1}$, J.D. Chen ${ }^{1}$ and \\ Q.Q. Zhang ${ }^{1,2}$ \\ ${ }^{1}$ Institute of Biomedical and Pharmaceutical Technology, Fuzhou University, \\ Fuzhou, China \\ ${ }^{2}$ Institute of Biomedical Engineering, Chinese Academy of Medical Sciences, \\ Tianjin, China
}

${ }^{*}$ These authors contributed equally to this study.

Corresponding authors: Z.L. Wang / Q.Q. Zhang

E-mail: IBPTwzl@fzu.edu.cn / zhangqiq@126.com

Genet. Mol. Res. 13 (3): 4883-4892 (2014)

Received March 26, 2013

Accepted November 20, 2013

Published July 4, 2014

DOI http://dx.doi.org/10.4238/2014.July.4.2

\begin{abstract}
In this study, two antioxidative substances, a homogeneous polysaccharide [abalone shell polysaccharide (ASP-1), corresponding to the first peak by size exclusion chromatography] and a non-polysaccharide compound [abalone shell compound (ACS-2), corresponding to the second peak by size exclusion chromatography], were extracted from the abalone (Haliotis discus hannai Ino) shell. We primarily focused on the investigation of ASP-1. As a heteropolysaccharide, ASP-1 is comprised of 9.3\% uronic acid and $86.4 \%$ saccharide, the latter including mannose, ribose, rhamnose, glucose, galactose, arabinose, and two unknown monosaccharides, NO1 and NO2, with a mass ratio of 9.5:10.1:2.2:18.2:21.8:5.5:16.5:16.2. The antioxidant activity assays indicated that $5.0 \mathrm{mg} / \mathrm{mL}$ ASP-1 has significant scavenging effects on superoxide radicals $(86.2 \%)$ compared to the positive control of ascorbic acid (95.6\%).
\end{abstract}

Key words: Abalone shell; Polysaccharides; Antioxidant activity 


\section{INTRODUCTION}

Antioxidants are widely used in food preservation and healthcare practices, as they are capable of eliminating reactive oxygen species (ROS), counteracting the damaging effects of oxidation, and prolonging the preservation time of foods. However, several reports have shown that traditional antioxidants, such as beta carotene, vitamin $\mathrm{A}$, and vitamin $\mathrm{E}$, may be detrimental to human health (Bjelakovic et al., 2007). Recently, considerable interest has arisen for the development of antioxidants from natural sources, such as marine flora and fauna, bacteria, fungi, and higher plants (Chattopadhyay et al., 2010).

The Pacific abalone, Haliotis discus hannai Ino, which is mainly distributed in East Asia (Marchant et al., 2009), is the most commercially important species of gastropods in aquaculture (Chen et al., 2005). Large amounts of abalone shell are discarded as waste during the processing of abalone. Since China is the largest abalone producer in the world, exploitation of deserted abalone shells can reduce the burden on the environment and generate added value.

In China, abalone shells are traditionally prescribed for treating glaucoma, headaches, and vertigo, based on the potential antioxidant activity of their soluble compositions (Zhou and $\mathrm{Wu}, 2001)$. Our previous studies demonstrated that the soluble matrix of abalone shell exhibited ferric reducing capacity and scavenging capacity of superoxide radicals $\left(\mathrm{O}_{2}^{-}\right)$similar to that of the pearl, while showing little or no inhibition of 1,1-diphenyl-2-picrylhydrazyl (DPPH) and hydroxyl (OH)-free radicals (Liang et al., 2010). Based on the above findings, the present study was designed to further investigate the antioxidative components isolated from the shell of H. discus hannai Ino, as few related studies are currently available.

In this study, a water-soluble polysaccharide, abalone shell polysaccharide (ASP-1), corresponding to the first peak by size exclusion chromatography (SEC) and a non-polysaccharide compound, abalone shell compound (ASC-2), corresponding to the second peak by SEC were extracted from the shell of abalone, and their capacities to scavenge the superoxide radical were assessed. Furthermore, the physicochemical properties of ASP-1 were analyzed.

\section{MATERIAL AND METHODS}

\section{Preparation of crude polysaccharides from abalone shell}

Shells of $H$. discus hannai Ino were purchased from the Yilai Abalone Products Company, Fujian Province, China. The shells were washed thoroughly with tap water, dried by forced air circulation, and ground to a $100-\mu \mathrm{m}$ particle size. A 1.2-kg shell powder sample was suspended in $7.2 \mathrm{~L}$ distilled water for $3 \mathrm{~h}$ at $80^{\circ} \mathrm{C}$ with continuous stirring, followed by filtration. The supernatant solution was obtained from centrifugation of the suspension at $8000 \mathrm{rpm}$ for $30 \mathrm{~min}$, which was then concentrated to approximately $500 \mathrm{~mL}$ under reduced pressure at $40^{\circ} \mathrm{C}$ using a rotary evaporator. The resultant solution was precipitated with ethanol up to $75 \%$ $(\mathrm{v} / \mathrm{v})$ and left at $4^{\circ} \mathrm{C}$ overnight. Finally, the precipitate was obtained by centrifugation and was dehydrated by air-drying to produce approximately $360 \mathrm{mg}$ dried powder.

The protein in the dried powder was removed using the trichloroacetic acid (TCA) precipitation method. The dried powder was redissolved in distilled water and then mixed with $10 \%$ TCA solution at a 2:1 (v/v) ratio. The mixture was oscillated intensively for $2 \mathrm{~h}$ at room temperature and then centrifuged at $10,000 \mathrm{rpm}$ for $15 \mathrm{~min}$ at $4^{\circ} \mathrm{C}$. The supernatant 
solution was dialyzed against $1 \mathrm{~L}$ ultrapure water five times using a 1-kDa molecular weight cut-off membrane to remove pigments, sugar residues, and other low molecular weight matter. Finally, the retentate was lyophilized, yielding $240 \mathrm{mg}$ crude polysaccharide.

\section{Purification of polysaccharides by SEC}

The crude polysaccharide was dissolved in $0.1 \mathrm{M} \mathrm{NaCl}$ solution to obtain a $10 \mathrm{mg} /$ $\mathrm{mL}$ final solution concentration. The resultant solution was applied to a $1.6 \mathrm{x} 80 \mathrm{~cm}$ Sephadex G-75 column (General Electric Company, USA) using $100 \mathrm{mM} \mathrm{NaCl}$ buffer as the solvent. Fractions were collected and analyzed for neutral sugar content by the phenol-sulfuric acid method (Masuko et al., 2005). Appropriate fractions (namely, ASP-1 and ASC-2, corresponding to the peaks in Figure 1) were pooled, dialyzed, and lyophilized.

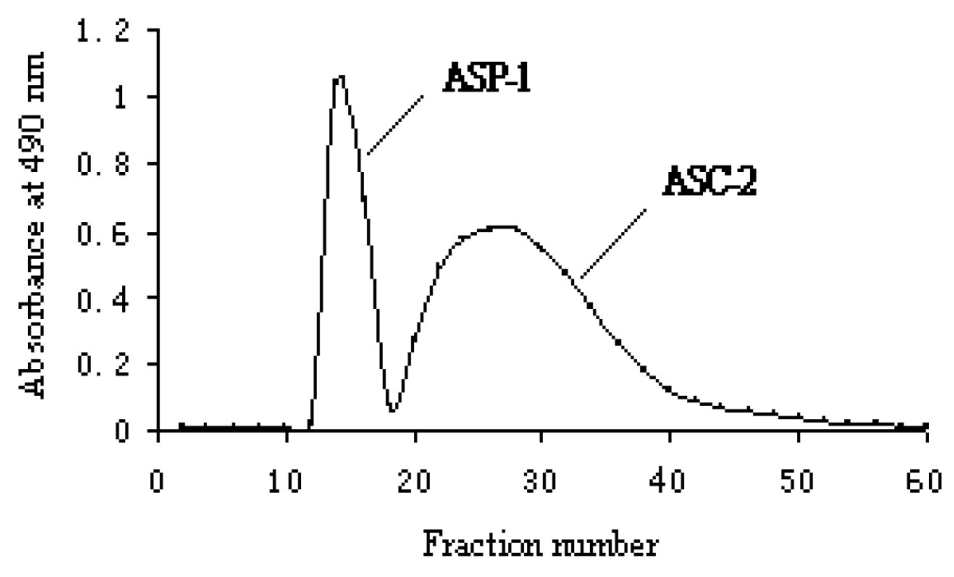

Figure 1. Chromatogram of crude polysaccharide from abalone shell on Sephadex G-75 column.

Ultraviolet-visible (UV/Vis) spectra of the sample (ASP-1) were recorded using a Varian Cary $50 \mathrm{UV} /$ Vis spectrophotometer (Tianjin, China). To determine the homogeneity of ASP-1, the sample was resolved on 1.0\% agarose gel by electrophoresis and stained with $0.1 \%$ toluidine blue in acetic acid:ethanol:water (0.1:5:5, v/v/v) (Volpi and Maccari, 2006).

\section{Determination of total sugar and uronic acid contents in ASP-1}

The total sugar content was determined by the phenol-sulfuric acid method, using glucose as the standard (Masuko et al., 2005). The uronic acid content was measured by the carbozole reaction with glucuronic acid (GlcUA) as the standard (Chaplin and Kennedy, 1986). The sulfate content was estimated by the turbidometric barium chloride method using potassium sulfate as the standard (Ghosh et al., 2004).

\section{Infrared (IR) analysis}

The IR spectra were obtained according to the method described by Kumar et al. 
(2004) in the 400 to $4000 \mathrm{~cm}^{-1}$ frequency range on a Fourier transform infrared (FTIR) Spectrometer (Nicolet 5700, Thermo Fisher Scientific, USA).

\section{Monosaccharide composition analysis}

Standard samples were prepared by following the method of Ling et al. (2008), with some modifications. A set of monosaccharide standards (Sigma, USA), $2.0 \mathrm{mg}$ of each mannose (Man), ribose (Rib), rhamnose (Rha), GlcUA, glucose (Glu), galactose (Gal), xylose (Xyl), and arabinose (Ara), were resolved in $1.0 \mathrm{~mL}$ distilled water. Twenty-five microliters resultant solution was labeled with 1-phenyl-3-methyl-5-pyrazolone (PMP) by adding $0.4 \mathrm{M}$ methanolic solution of $50 \mu \mathrm{L} \mathrm{PMP}$ and $25 \mu \mathrm{L} 0.6 \mathrm{M}$ sodium hydroxide, vortexed, and incubated at $70^{\circ} \mathrm{C}$ for $100 \mathrm{~min}$. The mixture was neutralized by adding $50 \mu \mathrm{L} 0.3 \mathrm{M}$ hydrochloric acid solution. Then, extraction treatment of the neutralized mixture with distilled water and chloroform was performed three or more times. The resulting aqueous phase was degassed and filtered through a $0.22-\mu \mathrm{m}$ polytetrafluoroethylene filter for high-performance liquid chromatography (HPLC) analysis.

One hundred microliters of $25 \mathrm{mg} / \mathrm{mL}$ ASP-1 solution was mixed with $150 \mu \mathrm{L} 2 \mathrm{M}$ trifluoroacetic acid at $110^{\circ} \mathrm{C}$ for $2 \mathrm{~h}$. Two hundred microliters of methanol was added into the mixture at room temperature and then dried by a stream of nitrogen. The product was treated by repeating the above process twice. The residue produced was dissolved in $50 \mu \mathrm{L}$ distilled water. Twenty-five microliters of residue solution was labeled with PMP in the same way as applied to the labeling of standard samples.

HPLC analysis was carried out on an analytical HPLC system (EasySep 1010, Unimicro Technologies Co. Ltd., USA) with a C18 column ( $4.6 \mathrm{~mm}$ in internal diameter x $250 \mathrm{~mm}$, $5 \mu \mathrm{m}$ octadecyl silane) and a Variable Wavelength UV Detector. An optimized program of isocratic elution at $30^{\circ} \mathrm{C}$ with a flow rate of $1.0 \mathrm{~mL} / \mathrm{min}$ was used. The mobile phase consisted of $83 \% 0.1 \mathrm{M}$ phosphate-buffered saline (PBS), $\mathrm{pH} 6.7$, and $13 \%$ acetonitrile. The isocratic elution program was run for $70 \mathrm{~min}$. The wavelength for UV detection was $254 \mathrm{~nm}$.

\section{Assay of $\mathrm{O}_{2}^{-\cdot}$ scavenging}

The $\mathrm{O}_{2}^{-\cdot}$ scavenging assay was based on the method of Moumen et al. (2001), with some modifications. The reaction mixture included: $1.0 \mathrm{~mL} 75 \mathrm{mM}$ PBS, $\mathrm{pH} 7.8,200 \mu \mathrm{L}$ ASP-1 solutions of different concentrations, $100 \mu \mathrm{L} 0.1 \mathrm{M}$ hydroxylamine hydrochloride, $100 \mu \mathrm{L} 0.75 \mathrm{mM}$ xanthine, and $100 \mu \mathrm{L} 0.1 \mathrm{U} / \mathrm{mL}$ xanthine oxidase. The mixture solution was incubated at $37^{\circ} \mathrm{C}$ for $30 \mathrm{~min}$. The chromogenic reaction was triggered by a color agent and processed at room temperature for $10 \mathrm{~min}$. The absorbance at $530 \mathrm{~nm}$ was recorded. The scavenging rate of superoxide radical production was calculated as follows:

$\mathrm{O}_{2}^{-\cdot}$ scavenging rate $(\%)=(\mathrm{Ao}-\mathrm{Ai}) / \mathrm{Ao} \times 100$, where Ao represents the absorbance of the control and Ai represents the absorbance in the presence of the sample.

\section{Statistical analysis}

All experiments were performed in triplicate. The results are reported as means $\pm \mathrm{SD}$. One-way analysis of variance was applied to determine differences in means. A P value $<0.05$ denoted statistical significance. 


\section{RESULTS AND DISCUSSION}

\section{Isolation and purification of ASP-1}

The crude polysaccharide obtained from the shells of abalone (H. discus hannai Ino) was ultimately separated into two main sub-fractions: $75.5 \mathrm{mg}$ ASP-1 and $57.6 \mathrm{mg}$ ASC-2 (Figure 1). In this study, we only focused on the characteristics and composition of ASP-1.

No absorption was observed at 260 and $280 \mathrm{~nm}$, indicating that the nucleic acids and proteins in ASP-1 were undetectable (Figure 2). ASP-1 resulted in a negative test with Fehling's solution. The homogeneity of the ASP-1 polysaccharide was illustrated by a single spot on the agarose gel and a highly symmetrical peak in gel-filtration chromatography (Figure 3 ), paving the way for further study of its characterization.

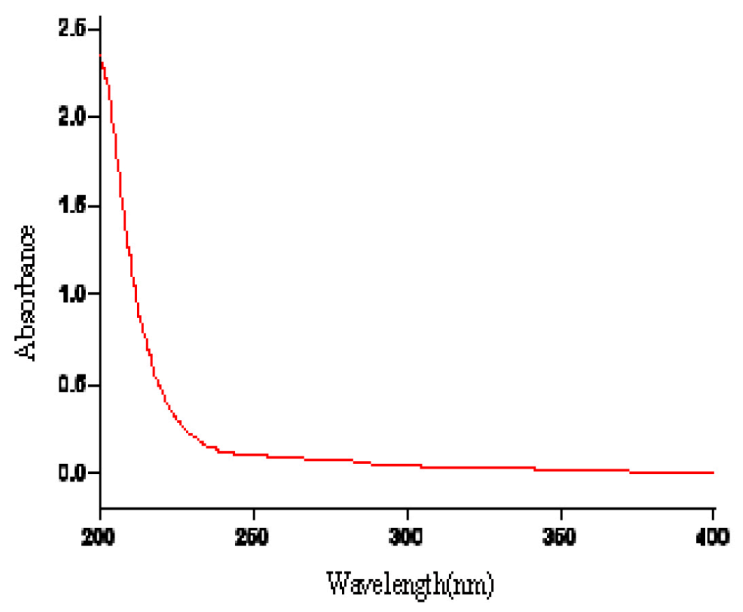

Figure 2. UV spectra of ASP-1 at 200-400 nm; the curve shows no clear peak.

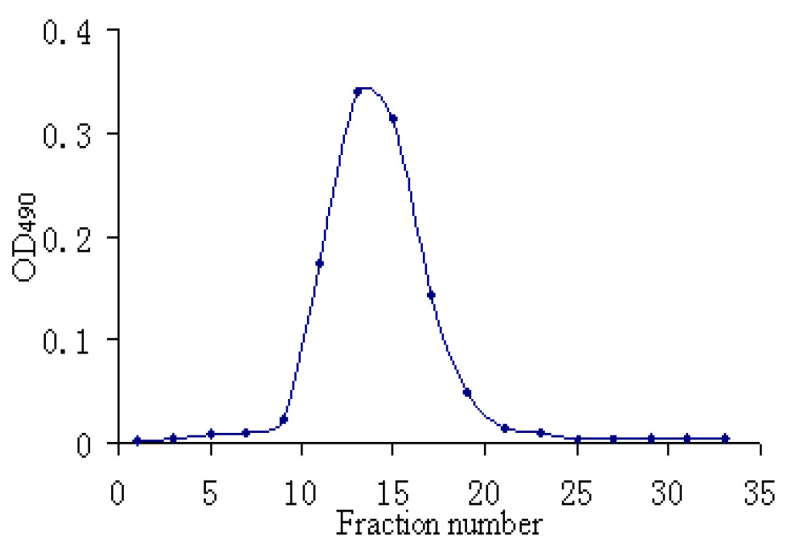

Figure 3. Chromatogram of ASP-1 on Sephadex G-75 column eluting with $0.1 \mathrm{M} \mathrm{NaCl}$ at a flow rate of $0.3 \mathrm{~mL} / \mathrm{min}$; the absorbance at $490 \mathrm{~nm}$ was that of the resulting reactive solutions of polysaccharides, phenol and sulfuric acid. 


\section{Carbohydrate and uronic acid contents of ASP-1}

The carbohydrate and uronic acid contents of the ASP-1 fraction are summarized in Table 1. ASP-1 had a high total carbohydrate content $(86.4 \pm 0.62 \%)$, whereas the uronic acid content in ASP-1 was only $9.3 \pm 0.24 \%$. In addition, sulfate groups, which are often present in polysaccharides isolated from marine organisms (Zhu et al., 2008a; Karmakar et al., 2009), were not found in ASP-1.

Table 1. Carbohydrate and uronic acid content of ASP-1 $(\mathrm{N}=3)$.
\begin{tabular}{|cccc}
\hline Sample & Carbohydrate $(\mathrm{w} \%)$ & Uronic acid $(\mathrm{w} \%)$ & Sulfated group $(\mathrm{w} \%)$ \\
\hline ASP-1 & $86.4 \pm 0.62$ & $9.3 \pm 0.24$ & - \\
\hline
\end{tabular}

\section{IR analysis of ASP-1}

The FTIR spectrum of ASP-1 is shown in Figure 4. The broad band at approximately $3401 \mathrm{~cm}^{-1}$ was assigned to $\mathrm{O}-\mathrm{H}$ stretching vibration. The characteristic band for $\mathrm{C}-\mathrm{H}$ appeared at $2934 \mathrm{~cm}^{-1}$. The characteristic band of carbonyl stretching appeared at $1650 \mathrm{~cm}^{-1}$ (Zheng et al., 2005). The absorption peaks at $1407 \mathrm{~cm}^{-1}$ and $1378 \mathrm{~cm}^{-1}$ corresponded to the stretching vibration of $\mathrm{C}-\mathrm{O}$ of uronic acid (Yang et al., 2008). The strong absorption at $1073 \mathrm{~cm}^{-1}$ was dominated by the glycosidic linkage $\mathrm{C}-\mathrm{O}-\mathrm{C}$ stretching vibration contribution (Yang et al., 2009). In addition, ASP-1 exhibited an absorption peak at $894 \mathrm{~cm}^{-1}$, which is the characteristic absorption of a $\beta$-anomeric configuration(Helm et al., 2000). The IR absorption of ASP-1 was at $809 \mathrm{~cm}^{-1}$, which corresponds to the characteristic absorption of man (Tao and Zhang, 2008).

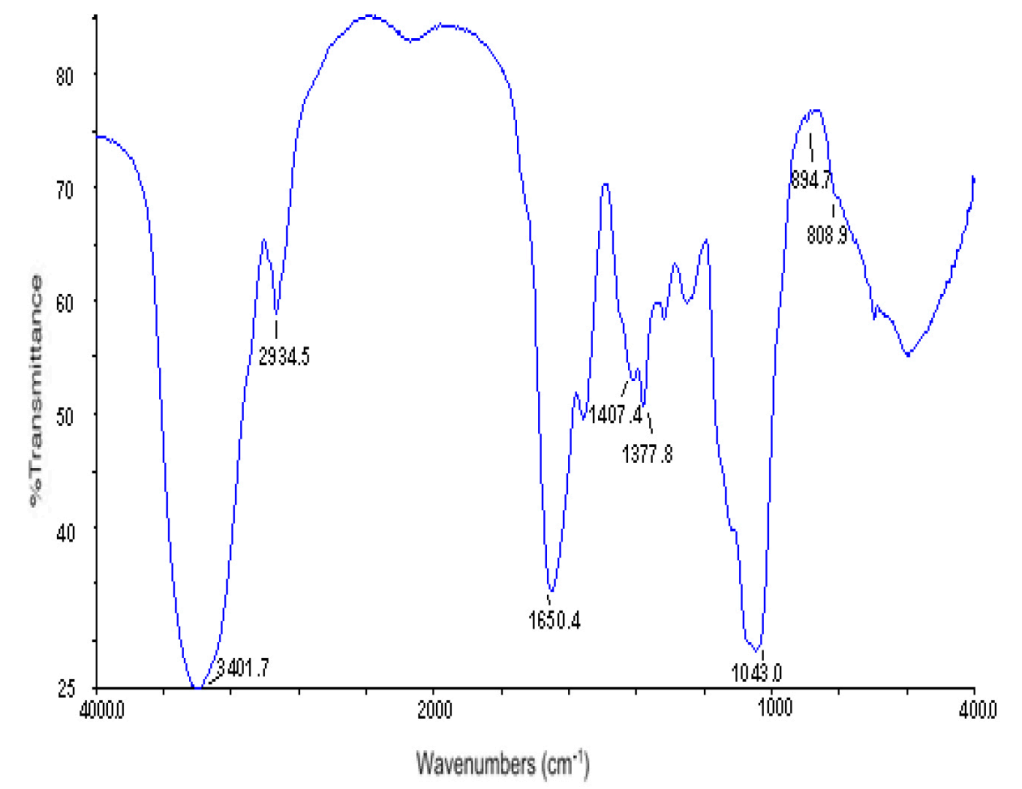

Figure 4. Fourier transform infrared spectra of ASP-1. 


\section{Carbohydrate composition of ASP-1}

An equal mass mixture of eight monosaccharides was labeled with PMP and separated by reverse-phase HPLC (Figure 5A). All eight PMP-labeled monosaccharides were clearly separated within $60 \mathrm{~min}$. The peaks for all monosaccharides were sharp and symmetrical. Excellent run-to-run reproducibility with respect to retention time was displayed.

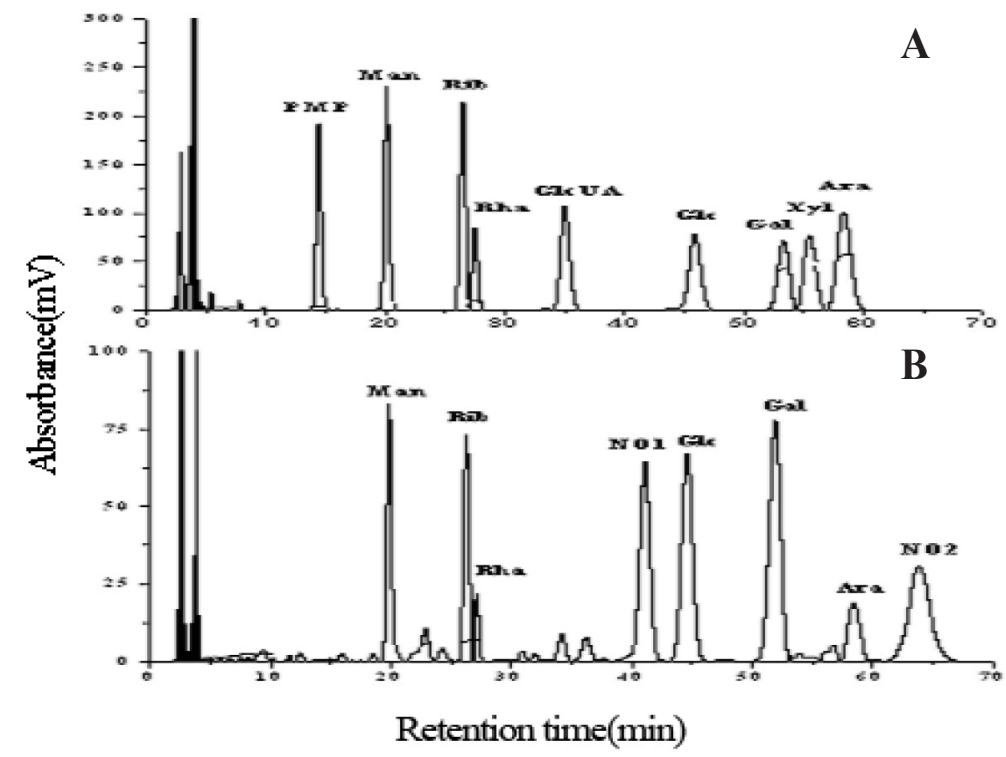

Figure 5. HPLC chromatograms of PMP derivatives of (A) 8 standard monosaccharides and (B) hydrolyzed ASP-1.

The results of the HPLC analysis of ASP-1 are shown in Figure 5B. By comparing the retention times of peaks to reference sugar standards and chromatography with added standards, six specific monosaccharides, including Man, Rib, Rha, Glu, Gal, Ara, and two unknown monosaccharides, $\mathrm{NO} 1$ and NO2, were identified with a mass ratio of 9.5:10.1:2.2: 18.2:21.8:5.5:16.5:16.2 (Table 2). This result clearly demonstrated that Gal was the dominant monosaccharide, whereas Rha was the most minor. The composition of ASP-1 differed from that of abalone polysaccharides previously reported (Zhu et al., 2008a,b).

Table 2. Monosaccharide composition of ASP-1.
\begin{tabular}{lccc}
\hline Monosaccharide & Retention time of standard monosaccharides/min & Retention time of hydrolysates/min & $\%$ \\
\hline Mannose & 20.047 & 19.815 & 9.5 \\
Ribose & 26.424 & 26.258 & 10.1 \\
Rhamnose & 27.429 & 27.043 & 2.2 \\
Glucuronic acid & 34.958 & - & - \\
NO1 & - & 41.061 & 16.5 \\
Glucose & 45.825 & 44.585 & 18.2 \\
Galactose & 52.231 & 51.894 & 21.8 \\
Xylose & 55.384 & - & - \\
Arabinose & 58.289 & 58.351 & 5.5 \\
NO2 & - & 63.788 & 16.2 \\
\hline
\end{tabular}




\section{Scavenging effects on superoxide radicals}

As shown in Figure 6, ASP-1 and ASC-2 showed a significant increase $(\mathrm{P}<0.01)$ in superoxide radical scavenging activity with increasing concentrations. In the $0.01-2.0 \mathrm{mg} / \mathrm{mL}$ concentration range, the $\mathrm{O}_{2}^{-} \cdot$ scavenging ability of ASP-1 increased from 1.88 to $80.4 \%$, and that of ASC-2 increased from 0.63 to $71.5 \%$ (Figure 6). At $5.0 \mathrm{mg} / \mathrm{mL}$, the superoxide radical scavenging rates of ASP-1 and ASC-2 reached 86.4 and $82.7 \%$, respectively. However, the scavenging activity of ascorbic acid $(\mathrm{Vc})$ on superoxide radicals was significantly $(\mathrm{P}<0.05)$ higher than those of ASP-1 and ASC-2. The $\mathrm{IC}_{50}$ (the antioxidant concentration value needed to scavenge half of the superoxide radicals) of $\mathrm{Vc}, \mathrm{ASP}-1$, and ASC-2 were approximately $0.045,0.4$, and $0.75 \mathrm{mg} / \mathrm{mL}$, respectively. The relationship between the scavenging rate of superoxide radicals (y) and ASP-1 or ASP-2 concentration (x) was found to exhibit the following logarithmic functions: for ASP-1, $\mathrm{y}=15.78 \ln (\mathrm{x})+64.81\left(\mathrm{R}^{2}=0.9538\right)$, and for ASC-2, $\mathrm{y}=$ $14.604 \ln (\mathrm{x})+56.435\left(\mathrm{R}^{2}=0.9496\right)$.

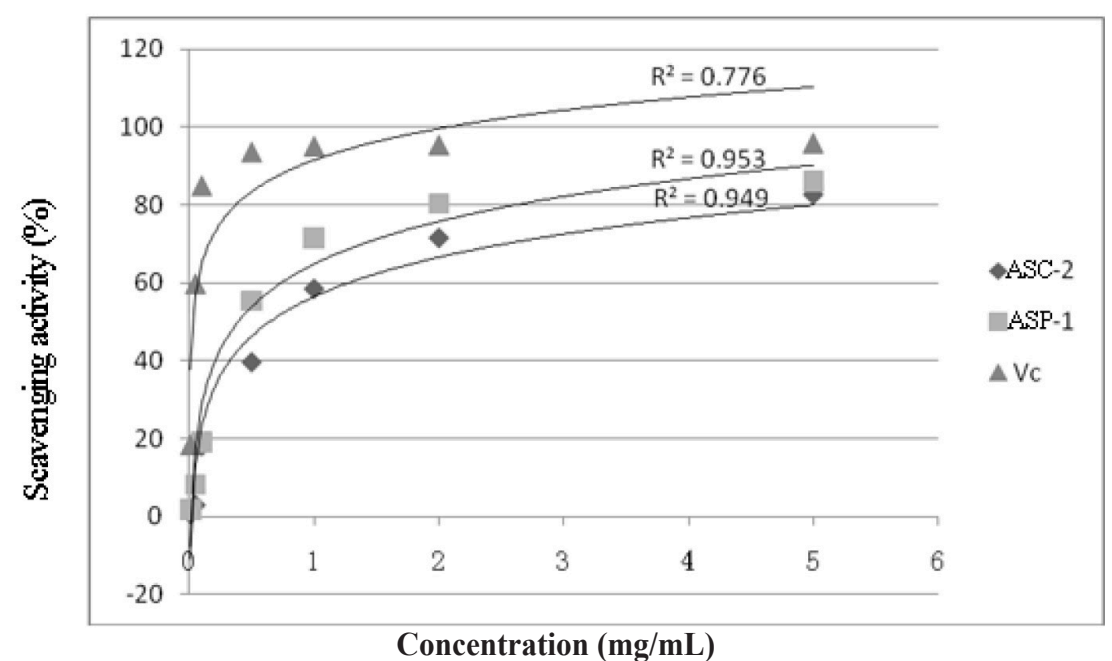

Figure 6. Scavenging activity of ASP-1 and ASC-2 against superoxide radical with ascorbic acid (Vc) as a positive control.

Superoxide anions play an important role in the formation of other ROS, or act as precursors to active free radicals, which results in oxidative damage to biological molecules such as lipids, proteins, and DNA (Halliwell and Gutteridge, 1984; Pietta, 2000; Wickens, 2001). ASP-1 and ASP-2 are able to inhibit the superoxide radicals generated by the xanthine $(\mathrm{X})$ /xanthine oxidase $(\mathrm{XO})$ system, exhibiting a great potential for combating oxidation. One possible superoxide radical scavenging mechanism of ASP-1 and ASC-2 is that they combine with superoxide radical ions to form a stable radical to terminate the radical chain reaction (Liu et al., 2007). It is worth noting that the characteristics of polysaccharides, i.e., sugar components, molecular weight, and the structure of the main chain and branches, strongly affect their antioxidant activities. However, the antioxidant mechanism-related characteristics of ASP-1 and ASC-2 remain unclear. 


\section{Concluding remarks}

We successfully isolated a crude polysaccharide fraction from the shells of abalone, $H$. discus hannai Ino, by hot water extraction. The fraction was purified using a Sephadex G-75 column, and produced two sub-fractions named ASP-1 and ASC-2. ASP-1 was shown to be a homogeneous polysaccharide by the UV/Vis spectrum, Sephadex G-75 column chromatography, and agarose gel electrophoresis. FTIR and quantitative carbohydrate composition analysis showed that the obtained ASP-1 is a $\beta$-type heteropolysaccharide, which is composed of Man, Rib, Rha, Glu, Gal, Ara, and two unknown monosaccharides, NO1 and NO2, at the mass ratio of 9.5:10.1:2.2:18.2:21.8:5.5:16.5:16.2. ASP- 1 was composed of $86.4 \pm 0.62 \%$ carbohydrate and $9.3 \pm 0.62 \%$ uronic acid. However, quantifying the total sugar of ASP-1, which comprises several kinds of monocarbohydrates, with only D-glucuronic acid as the standard may result in some deviations from the true value. A dose-dependent relationship was found between the antioxidant capability and concentration of ASP-1. Sulfate groups of polysaccharides are known to have a significant effect on antioxidant activity (Tsiapali et al., 2001; Zhao et al., 2004). Yet, such sulfate groups were not detected in ASP-1 samples. To the best of our knowledge, this is a unique antioxidant polysaccharide that has never been reported.

In addition, ASC-2 might be an antioxidant compound without a saccharide, given that no spot was observed on the agarose gel, no monosaccharide was detected by the HPLC assay, and the asymmetrical broad peak corresponding to ASC-2 appeared in the size exclusion chromatographic curve.

Finally, this study clearly demonstrated that a polysaccharide was one of the main active ingredients in abalone shell, and provided basic information on purified ASP-1 polysaccharides from the shell of abalone. However, to date, it has not been possible to illustrate the antioxidant mechanism of ASP-1 in vitro. Interest in investigating other potential bioactivity and structure activity relationships of this macromolecule is expected to grow in years to come. Further study on ASP-1 may be of great help to promote the modernization of abalone shells used in traditional Chinese medicine.

\section{ACKNOWLEDGMENTS}

Research supported by the Key Project of the Fujian Provincial Department of Ocean and Fisheries (Contract \#[2008]1-12) and the Open Measuring Fund of Precious Apparatus of Fuzhou University (2012). We are grateful to Dr. Shengxiong Dong for helpful discussions.

\section{REFERENCES}

Bjelakovic G, Nikolova D, Gluud LL, Simonetti RG, et al. (2007). Mortality in randomized trials of antioxidant supplements for primary and secondary prevention: systematic review and meta-analysis. JAMA 297: 842-857.

Chaplin MF and Kennedy JF (1986). Uronic Acid (Carbazole) Assay. In: Carbohydrate Analysis - A Practical Approach. IRL Press, Oxford.

Chattopadhyay N, Ghosh T, Sinha S, Chattopadhyay K, et al. (2010). Polysaccharides from Turbinaria conoides: Structural features and antioxidant capacity. Food Chem.118: 823-829.

Chen H, Mai K, Zhang W, Liufu Z, et al. (2005). Effects of dietary pyridoxine on immune responses in abalone, Haliotis discus hannai Ino. Fish Shellfish Immunol. 19: 241-252.

Ghosh P, Adhikari U, Ghosal PK, Pujol CA, et al. (2004). In vitro anti-herpetic activity of sulfated polysaccharide fractions from Caulerpa racemosa. Phytochemistry 65: 3151-3157. 
Halliwell B and Gutteridge JM (1984). Lipid peroxidation, oxygen radicals, cell damage, and antioxidant therapy. Lancet 1: 1396-1397.

Helm RF, Huang Z, Edwards D, Leeson H, et al. (2000). Structural characterization of the released polysaccharide of desiccation-tolerant Nostoc commune DRH-1. J. Bacteriol. 182: 974-982.

Karmakar P, Ghosh T, Sinha S, Saha S, et al. (2009). Polysaccharides from the brown seaweed Padina tetrastromatica: Characterization of a sulfated fucan. Carbohydr. Polym. 78: 416-421.

Kumar CG, Joo HS, Choi JW, Koo YM. et al. (2004). Purification and characterization of of an extracellular polysaccharide from haloalkalophilic Bacillus sp. I-450. Enzyme Microb. Technol. 34: 673-681.

Liang HB, Guo W, Wu JL and Peng ZF (2010). Study on antioxidant activity of water soluble matrix from abalone shell. Chin. J. Food Sci. 31: 113-116.

Ling Y, Wang YF, Wang Y, Xu X, et al. (2008). Determination of monosaccharide compostion in Cordyceps sinensis polysaccharide by HPLC with pre-column derivatization. Chin. J. Pharm. Aceuticals 39: 924-929.

Liu CH, Wang CH, Xu ZL and Wang Y (2007). Isolation, chemical characterization and antioxidant activities of two polysaccharides from the gel and the skin of Aloe barbadensis Miller irriagted with sea water. Process Biochem. 42: 961-970.

Marchant S, Haye PA, Marín SA and Winkler FM (2009). Genetic variability revealed with microsatellite markers in an introduced population of the abalone Haliotis discus hannai Ino. Aquaculture Res. 40: 298-304.

Masuko T, Minami A, Iwasaki N, Majima T, et al. (2005). Carbohydrate analysis by a phenol-sulfuric acid method in microplate format. Anal. Biochem. 339: 69-72.

Moumen R, Ait-Oukhatar N, Bureau F, Fleury C, et al. (2001). Aluminium increases xanthine oxidase activity and disturbs antioxidant status in the rat. J. Trace Elem. Med. Biol. 15: 89-93.

Pietta PG (2000). Flavonoids as antioxidants. J. Nat. Prod. 63: 1035-1042.

Tao Y and Zhang L (2008). Characterization of polysaccharide-protein complexes by size-exclusion chromatography combined with three detectors. Carbohydr. Res. 343: 2251-2257.

Tsiapali E, Whaley S, Kalbfleisch J, Ensley HE, et al. (2001). Glucans exhibit weak antioxidant activity, but stimulate macrophage free radical activity. Free Radic. Biol. Med. 30: 393-402.

Volpi N and Maccari F (2006). Electrophoretic approaches to the analysis of complex polysaccharides. J. Chromatogr. B Analyt. Technol. Biomed. Life Sci. 834: 1-13.

Wickens AP (2001). Ageing and the free radical theory. Respir. Physiol. 128: 379-391.

Yang B, Jiang YM, Zhao M, Chen F, et al. (2009). Structural characterisation of polysaccharides purified from longan (Dimocarpus longan Lour) fruit of pericarp. Food Chem. 115: 609-614.

Yang C, He N, Ling X, Ye M, et al. (2008). The isolation and characterization of polysaccharides from longan pulp. Sep. Purif. Technol. 63: 226-230.

Zhao X, Xue CH, Li ZJ, Cai YP, et al. (2004). Antioxidant and hepatoprotective activities of low molecular weight sulfated polysaccharide from Laminaria japonica. J. Appl. Phycol. 16: 111-115.

Zheng R, Jie S, Hanchuan D and Moucheng W (2005). Characterization and immunomodulating activities of polysaccharide from Lentinus edodes. Int. Immunopharmacol. 5: 811-820.

Zhou DX and Wu SL (2001). Anti-inflammation and antioxidation of pearl water extract. J. Zhejiang Coll. TCM 25: 41-42.

Zhu BW, Yang JF, Li DG and Sun LM (2008a). Structure investigation of polysaccharide from abalone, Haliotis discus hannai Ino viscera. J. Biotechnol. 136: 591-592.

Zhu BW, Wang LS, Zhou DY, Li DM, et al. (2008b). Antioxidant activity of sulphated polysaccharide conjugates from abalone (Haliotis discus hannai Ino). Eur. Food Res. Technol. 227: 1663-1668. 